\title{
Stability of the Stochastic Reaction-Diffusion Neural Network with Time-Varying Delays and $p$-Laplacian
}

\author{
Pan Qingfei, ${ }^{1}$ Zhang Zifang, $^{2}$ and Huang Jingchang ${ }^{3}$ \\ ${ }^{1}$ College of Civil Engineering and Architecture, Sanming University, Sanming 365004, China \\ ${ }^{2}$ Department of Mathematics and Physics, Huaihai Institute of Technology, Lianyungang 222005, China \\ ${ }^{3}$ Science and Technology on Microsystems Laboratory, Shanghai Institute of MicroSystems and Information \\ Technology, CAS, Shanghai 200050, China \\ Correspondence should be addressed to Pan Qingfei, pqf101@yahoo.com.cn
}

Received 22 October 2012; Accepted 9 November 2012

Academic Editor: Maoan Han

Copyright (C) 2012 Pan Qingfei et al. This is an open access article distributed under the Creative Commons Attribution License, which permits unrestricted use, distribution, and reproduction in any medium, provided the original work is properly cited.

\begin{abstract}
The main aim of this paper is to discuss moment exponential stability for a stochastic reactiondiffusion neural network with time-varying delays and $p$-Laplacian. Using the Itô formula, a delay differential inequality and the characteristics of the neural network, the algebraic conditions for the moment exponential stability of the nonconstant equilibrium solution are derived. An example is also given for illustration.
\end{abstract}

\section{Introduction}

In many neural networks, time delays cannot be avoided. For example, in electronic neural networks, time delays will be present due to the finite switching speed of amplifies. In fact, time delays are often encountered in various engineering, biological, and economical systems. On the other hand, when designing a neural network to solve a problem such as optimization or pattern recognition, we need foremost to guarantee that the neural networks model is globally asymptotically stable. However, the existence of time delay frequently causes oscillation, divergence, or instability in neural networks. In recent years, the stability of neural networks with delays or without delays has become a topic of great theoretical and practical importance (see [1-16]).

The stability of neural networks which depicted by partial differential equations was studied in $[6,7]$. Stochastic differential equations were employed to research the stability of neural networks in [8-11], while [12, 13] used stochastic partial differential 
equations to analysis this question. In [15], the authors studied almost exponential stability for a stochastic recurrent neural network with time-varying delays. In addition, moment exponential stability for a stochastic reaction-diffusion neural network with time-varying delays is discussed in [16].

In this paper, we consider the stochastic reaction-diffusion neural network with timevarying delays and $p$-Laplacian as follows:

$$
\begin{aligned}
& d u_{i}(t, x)= {\left[\sum_{k=1}^{m} \frac{\partial}{\partial x_{k}}\left(\left|\nabla u_{i}\right|^{p-2} \frac{\partial u_{i}}{\partial x_{k}}\right)-a_{i}(u) u_{i}+I_{i}+\sum_{j=1}^{n} T_{i j} g_{j}\left(u_{j}\left(t-\tau_{j}(t), x\right)\right)\right] d t } \\
&+\sum_{l=1}^{m} \sigma_{i l}\left(u_{i}(t, x)\right) d W_{l}(t), \quad i=1,2, \ldots, n, t>t_{0}, x \in \Omega \\
& \frac{\partial u_{i}}{\partial n}:=\left(\frac{\partial u_{i}}{\partial x_{1}}, \ldots, \frac{\partial u_{i}}{\partial x_{m}}\right)^{T}=0, \quad i=1,2, \ldots, n, t \geq t_{0}, x \in \partial \Omega \\
& u_{i}\left(t_{0}+s, x\right)=\phi_{i}(s, x), \quad-\tau_{i}\left(t_{0}\right) \leq s \leq 0,0 \leq \tau_{i}(t) \leq \tau_{i}, i=1,2, \ldots, n, x \in \Omega .
\end{aligned}
$$

In (1.1), $\nabla u_{i}=\left(\partial u_{i} / \partial x_{1}, \ldots, \partial u_{i} / \partial x_{m}\right)^{T}, p \geq 2$ is a common number. $\Omega \subseteq R^{m}$ is a bounded convex domain with smooth boundary $\partial \Omega$ and measure mes $\Omega>0$. $n$ denotes the numbers of neurons in the neural network, $u_{i}(t, x)$ corresponds to the state of the $i$ th neurons at time $t$ and in space $x$, the $a_{i}(u)$ is an amplification function. $I_{i}$ is output. $g_{j}\left(u_{j}\left(t-\tau_{j}, x\right)\right)$ denotes the output of the $j$ th neuron at time $t-\tau_{j}$ and in space $x$, namely, activation function which shows how neurons respond to each other. $W(t)=\left(W_{1}(t), \ldots, W_{m}(t)\right)^{T}$ is an m-dimensional Brownian motion which is defined on a complete probability space $(\mathcal{S}, \mathcal{F}, D)$ with a natural filtration $\left\{\mathcal{F}_{t}\right\}_{t \geq t_{0}}$ (i.e., $\left.\mathcal{F}_{t}=\sigma\left\{W(s): t_{0} \leq s \leq t\right\}\right) . \sigma(u)=\left(\sigma_{1}\left(u_{1}\right), \ldots, \sigma_{n}\left(u_{n}\right)\right)^{T}, \sigma_{i}\left(u_{i}\right)=$ $\left(\sigma_{i 1}\left(u_{i}\right), \ldots, \sigma_{i m}\left(u_{i}\right)\right) . \sigma_{i j}\left(u_{i}\right)$ denotes the intensity of the stochastic perturbation. Functions $\sigma_{i j}\left(u_{i}\right)$ and $g_{i}$ are subject to certain conditions to be specified later. $T:=\left(T_{i j}\right)_{n \times n}$ is a real constant matrix and represents weight of the neuron interconnections, namely, $T_{i j}$ denotes the strength of $j$ th neuron on the $i$ th neuron at time $t-\tau_{j}$ and in space $x$, and $\tau_{j} \in[0, \tau]$ corresponds to axonal signal transmission delay.

\section{Definitions and Lemmas}

Throughout this paper, unless otherwise specified, let $|\cdot|$ denote Euclidean norm. Define that $|x|^{p}=\left(\sum_{i=1}^{n}\left|x_{i}\right|^{2}\right)^{p / 2}$ and $|x|_{p}=\sum_{i=1}^{n}\left|x_{i}\right|^{p}$ where $x=\left(x_{1}, \ldots, x_{n}\right)^{T} \in R^{n}$. Denote by $C([-\tau, 0] \times$ $\left.\Omega ; R^{n}\right)$ the family of continuous functions $\varphi$ from $[-\tau, 0] \times \Omega$ to $R^{n}$. For every $t \geq t_{0}$ and $p \geq 2$, denote by $L_{\mathscr{q}_{t}}^{p}\left([-\tau, 0] \times \Omega ; R^{n}\right)$ the family of all $\mathcal{F}_{t}$-measurable $C\left([-\tau, 0] \times \Omega ; R^{n}\right)$ valued random variables such that $\|\phi\|_{L^{p}}^{p}=\sup _{-\tau \leq \theta \leq 0} E\left(\|\phi(\theta)\|_{p}^{p}\right)<\infty$, where $\|\phi(\theta)\|_{p}=\left(\int_{\Omega}|\phi(\theta, x)|^{p} d x\right)^{1 / p}$, $E(\phi)$ denotes the expectation of random variable $\phi$.

Definition 2.1. The $u(t, x)=\left(u_{1}(t, x), \ldots, u_{n}(t, x)\right)^{T}$ is called a solution of problem (1.1)-(1.3) if it satisfies following conditions (1), (2), and (3):

(1) $u(t, x)$ adapts $\left\{\mathcal{F}_{t}\right\}_{t \geq t_{0}}$;

(2) for $T \in R_{t_{0}}^{+}:=\left\{t \in R: t \geq t_{0}\right\}, u(t, x) \in C\left(\left[t_{0}, T\right] \times \Omega, R^{n}\right)$, and $E\left(\max _{x \in \Omega} \int_{t_{0}}^{T}\left[|u(t, x)|^{p}+\right.\right.$ $\left.\left.|\nabla u(t, x)|^{p}\right] d t\right)<+\infty$, where $\nabla u(x, t)=\left(\partial u / \partial x_{1}, \ldots, \partial u / \partial x_{n}\right)$; 
(3) for $T \in R_{t_{0}}^{+}, t \in\left(t_{0}, T\right]$, it holds that

$$
\begin{gathered}
\int_{\Omega} u_{i}(t, x) d x=\int_{\Omega} \phi_{i}\left(t_{0}, x\right) d x+\int_{\Omega} \int_{t_{0}}^{t}\left\{\sum_{k=1}^{m} \frac{\partial}{\partial x_{k}}\left(\left|\nabla u_{i}\right|^{p-2} \frac{\partial u_{i}}{\partial x_{k}}\right)-a_{i}(u) u_{i}+I_{i}\right. \\
\left.+\sum_{j=1}^{n} T_{i j} g_{j}\left(u_{j}\left(\xi-\tau_{j}(\xi), x\right)\right)\right\} d \xi d x \\
+\sum_{l=1}^{m} \int_{\Omega} \int_{t_{0}}^{t} \sigma_{i l}\left(u_{i}(\xi, x)\right) d W_{l}(\xi) d x, \\
i=1, \ldots, n, \quad(t, x) \in\left(t_{0}, T\right] \times \Omega, \quad P \text { a.s., } \\
u_{i}\left(t_{0}+s, x\right)=\phi_{i}(s, x), \quad-\tau_{i} \leq s \leq 0, i=1, \ldots, n, P \text { a.s. }
\end{gathered}
$$

Definition 2.2. The $u=u^{*}(x)$ is called a nonconstant equilibrium solution of problem (1.1)(1.3) if and only if $u=u^{*}(x)$ satisfies (1.1) and (1.2).

Definition 2.3. The nonconstant equilibrium solution $u^{*}(x)$ of (1.1) about the given norm $\|\cdot\|_{\Omega}$ is called exponential stability in $p$ th moment, if there are constants $M>0, \delta>0$ for every stochastic field solution $u(t, x)$ of problem (1.1)-(1.3) such that

$$
E\left[\left\|u(t, x)-u_{*}\right\|_{\Omega}\right] \leq M e^{-\delta\left(t-t_{0}\right)},
$$

namely,

$$
\limsup _{t \rightarrow \infty} \frac{1}{t} \log E\left[\left\|u(t, x)-u_{0}\right\|_{\Omega}\right] \leq-\delta .
$$

The constant $-\delta$ on the right hand side in (2.3) is called Lyapunov exponent of every solution of problem (1.1)-(1.3) converging on equilibrium about norm $\|\cdot\|_{\Omega}$.

In order to obtain $p$ th moment exponential stability for a nonconstant equilibrium solution of problem (1.1)-(1.3), we need the following lemmas.

Lemma 2.4 (see [17]). Let $P=\left(p_{i j}\right)_{n \times n}$ and $Q=\left(q_{i j}\right)_{n \times n}$ be two real matrices. The continuous function $u_{i}(t) \geq 0$ satisfies the delay differential inequalities

$$
D^{+} u_{i}(t) \leq \sum_{j=1}^{n}\left[p_{i j} u_{j}(t)+q_{i j} u_{j}\left(t-\tau_{j}(t)\right)\right], \quad 0 \leq \tau_{i}(t) \leq \tau, i=1, \ldots, n .
$$

If $p_{i j}>0$ for $i \neq j$ and $q_{i j} \geq 0(i, j=1,2, \ldots, n)$ and $-(P+Q)$ is an M-matrix, then there are constants $k_{i}>0, \alpha>0$ such that

$$
u_{i}(t) \leq k_{i}\left(\sum_{j=1}^{n}\left\|\phi_{j}\right\|\right) \exp \left(-\alpha\left(t-t_{0}\right)\right), \quad i=1, \ldots, n, t \geq t_{0},
$$

where $\phi=\left(\phi_{1}, \ldots, \phi_{1}\right)$ are initial functions. $D^{+}$is right-hand upper derivate. $\|\cdot\|$ represents a norm. 
Lemma 2.5 (see [10]). Let $p>2$, then there are positive constants $e_{p}(n)$ and $d_{p}(n)$ for any $x=$ $\left(x_{1}, \ldots, x_{n}\right)^{T} \in R^{n}$ such that

$$
e_{p}(n)\left(\sum_{i=1}^{n}\left|x_{i}\right|^{2}\right)^{p / 2} \leq \sum_{i=1}^{n}\left|x_{i}\right|^{p} \leq d_{p}(n)\left(\sum_{i=1}^{n}\left|x_{i}\right|^{2}\right)^{p / 2} .
$$

Remark 2.6. If $p=2$, Lemma 2.5 also holds with $e_{p}(n)=d_{p}(n)=1$.

Suppose that $\sigma_{i j}\left(u_{i}\right), a_{i}(u)$, and $g_{i}$ are Lipschitz continuous such that the following conditions hold:

(H1) $\left|\left(\sigma_{i}\left(v_{1}\right)-\sigma_{i}\left(v_{2}\right)\right)\left(\sigma_{i}\left(v_{1}\right)-\sigma_{i}\left(v_{2}\right)\right)^{T}\right| \leq 2 \lambda_{i}\left|v_{1}-v_{2}\right|^{2}, i=1,2, \ldots, n$,

(H2) $\left|g_{i}\left(v_{1}\right)-g_{i}\left(v_{2}\right)\right| \leq c_{i}\left|v_{1}-v_{2}\right|, i=1,2, \ldots, n$,

(H3) $(u-v)\left(a_{i}(u) u-a_{i}(v) v\right) \geq a_{i}|u-v|^{2}$, for all $u, v \in R, i=1,2, \ldots, n$,

where $c_{i}, a_{i}$, and $\lambda_{i}(1 \leq i \leq n)$ are positive constants.

\section{Main Result}

Set $u(t, x)=\left(u_{1}(t, x), \ldots, u_{n}(t, x)\right)^{T}$ as a solution of the problem (1.1)-(1.3) and $u^{*}(x)=$ $\left(u_{1}^{*}(x), \ldots, u_{n}^{*}(x)\right)^{T}$ as a nonconstant equilibrium solution of the problem (1.1)-(1.3).

Theorem 3.1. Let $p \geq 2$ and (H1)-(H3) hold. Assume that there are positive constants $\varepsilon_{1}, \ldots, \varepsilon_{n}$ such that the matrix $M_{p}=-\left(p_{j i}+q_{i j}\right)_{n \times n}:=P^{T}+Q$ is an M-matrix, where

$$
\begin{gathered}
p_{i j}=-p \delta_{i j} a_{i}+p(p-1) \delta_{i j} \lambda_{i}+(p-1)\left|T_{i j}\right| c_{j} \varepsilon_{i}, \quad \delta_{i i}=1, \delta_{i j}=0(i \neq j), i, j=1, \ldots, n, \\
q_{i j}=\left|T_{i j}\right| c_{j} \varepsilon_{i}^{1-p}, \quad i, j=1, \ldots, n
\end{gathered}
$$

then the nonconstant equilibrium solution of problem (1.1)-(1.3) about $L^{p}$ norm is exponential stability in pth moment, that is, there are constants $C_{1}>0$ and $\alpha>0$, for any $t_{0} \in R^{+}$and any $\phi \in L_{F_{t_{0}}}^{p}$ such that

$$
E\left(\left\|u(t, x)-u^{*}\right\|_{p}^{p}\right) \leq C_{1} e^{-\alpha\left(t-t_{0}\right)}, \quad t \geq t_{0}
$$

Proof. Set $V_{i}(u(t, x))=\left|u_{i}(t, x)-u_{i}^{*}(x)\right|^{p}$. For every $t \geq t_{0}$ and $d t>0$, by means of Itô formula and (H3), one has that

$$
\begin{aligned}
d V_{i}(u)=p\left|u_{i}-u_{i}^{*}\right|^{p-2}\left(u_{i}-u_{i}^{*}\right)\{ & \sum_{k=1}^{m}\left[\frac{\partial}{\partial x_{k}}\left(\left|\nabla u_{i}\right|^{p-2} \frac{\partial u_{i}}{\partial x_{k}}\right)-\frac{\partial}{\partial x_{k}}\left(\left|\nabla u_{i}^{*}\right|^{p-2} \frac{\partial u_{i}^{*}}{\partial x_{k}}\right)\right] \\
& -\left(a_{i}(u) u_{i}-a_{i}\left(u^{*}\right) u_{i}^{*}\right) \\
& \left.+\sum_{j=1}^{n} T_{i j}\left(g_{j}\left(u_{j}\left(t-\tau_{j}, x\right)\right)-g_{j}\left(u_{j}^{*}\right)\right)\right\} d t
\end{aligned}
$$


Journal of Applied Mathematics

$$
\begin{aligned}
& \quad+\frac{1}{2} p(p-1)\left|u_{i}-u_{i}^{*}\right|^{p-2} \operatorname{sgn}\left(u_{i}-u_{i}^{*}\right)\left(\sigma_{i}\left(u_{i}\right)-\sigma_{i}\left(u_{i}^{*}\right)\right)\left(\sigma_{i}\left(u_{i}\right)-\sigma_{i}\left(u_{i}^{*}\right)\right)^{T} d t \\
& +p\left|u_{i}-u_{i}^{*}\right|^{p-2}\left(u_{i}-u_{i}^{*}\right)\left(\sigma_{i}\left(u_{i}\right)-\sigma_{i}\left(u_{i}^{*}\right)\right) d W(t) \\
& \leq p \sum_{k=1}^{m}\left|u_{i}-u_{i}^{*}\right|^{p-2}\left(u_{i}-u_{i}^{*}\right)\left[\frac{\partial}{\partial x_{k}}\left(\left|\nabla u_{i}\right|^{p-2} \frac{\partial u_{i}}{\partial x_{k}}\right)-\frac{\partial}{\partial x_{k}}\left(\left|\nabla u_{i}^{*}\right|^{p-2} \frac{\partial u_{i}^{*}}{\partial x_{k}}\right)\right] d t \\
& +\left(-p a_{i}+p(p-1) \lambda_{i}\right)\left|u_{i}-u_{i}^{*}\right|^{p} d t \\
& +p \sum_{j=1}^{n}\left|T_{i j}\right| c_{j}\left|u_{i}-u_{i}^{*}\right|^{p-2}\left|u_{i}-u_{i}^{*}\right|\left|u_{j}\left(t-\tau_{j}, x\right)-u_{j}^{*}\right| d t \\
& +p\left|u_{i}-u_{i}^{*}\right|^{p-2}\left(u_{i}-u_{i}^{*}\right)\left(\sigma_{i}\left(u_{i}\right)-\sigma_{i}\left(u_{i}^{*}\right)\right) d W(t) .
\end{aligned}
$$

Both sides of Inequality (3.3) are integrated about $x$ over $\Omega$. Set $\overline{V_{i}(u)}=\int_{\Omega} V_{i}(u) d x=\left\|u_{i}-u_{i}^{*}\right\|_{p}^{p}$. One has that

$$
\begin{aligned}
d \overline{V_{i}(u)} \leq & p \sum_{k=1}^{m} \int_{\Omega}\left|u_{i}-u_{i}^{*}\right|^{p-2}\left(u_{i}-u_{i}^{*}\right)\left[\frac{\partial}{\partial x_{k}}\left(\left|\nabla u_{i}\right|^{p-2} \frac{\partial u_{i}}{\partial x_{k}}\right)-\frac{\partial}{\partial x_{k}}\left(\left|\nabla u_{i}^{*}\right|^{p-2} \frac{\partial u_{i}^{*}}{\partial x_{k}}\right)\right] d x d t \\
& +\left(-p a_{i}+p(p-1) \lambda_{i}\right) \int_{\Omega}\left|u_{i}-u_{i}^{*}\right|^{p} d x d t \\
& +p \sum_{j=1}^{n} \int_{\Omega}\left|u_{i}-u_{i}^{*}\right|^{p-2}\left|u_{i}-u_{i}^{*}\right|\left|T_{i j}\right| c_{j}\left|u_{j}\left(t-\tau_{j}, x\right)-u_{j}^{*}\right| d x d t \\
& +p \sum_{l=1}^{m} \int_{\Omega}\left|u_{i}-u_{i}^{*}\right|^{p-2}\left(u_{i}-u_{i}^{*}\right)\left(\sigma_{i l}\left(u_{i}\right)-\sigma_{i l}\left(u_{i}^{*}\right)\right) d x d W_{l}(t) .
\end{aligned}
$$

Set $\left(\left|\nabla u_{i}\right|^{p-2}\left(\partial u_{i} / \partial x_{k}\right)-\left|\nabla u_{i}^{*}\right|^{p-2}\left(\partial u_{i}^{*} / \partial x_{k}\right)\right)_{k=1}^{m}:=\left(\left|\nabla u_{i}\right|^{p-2}\left(\partial u_{i} / \partial x_{1}\right)-\left|\nabla u_{i}^{*}\right|^{p-2}\left(\partial u_{i}^{*} / \partial x_{1}\right), \ldots\right.$, $\left.\left|\nabla u_{i}\right|^{p-2}\left(\partial u_{i} / \partial x_{m}\right)-\left|\nabla u_{i}^{*}\right|^{p-2}\left(\partial u_{i}^{*} / \partial x_{m}\right)\right)$. By (1.2), one has that

$$
\begin{aligned}
\sum_{k=1}^{m} \int_{\Omega}\left|u_{i}-u_{i}^{*}\right|^{p-2}\left(u_{i}-u_{i}^{*}\right)\left[\frac{\partial}{\partial x_{k}}\left(\left|\nabla u_{i}\right|^{p-2} \frac{\partial u_{i}}{\partial x_{k}}\right)-\frac{\partial}{\partial x_{k}}\left(\left|\nabla u_{i}^{*}\right|^{p-2} \frac{\partial u_{i}^{*}}{\partial x_{k}}\right)\right] d x \\
=\int_{\Omega}\left|u_{i}-u_{i}^{*}\right|^{p-2}\left(u_{i}-u_{i}^{*}\right) \nabla \cdot\left(\left|\nabla u_{i}\right|^{p-2} \frac{\partial u_{i}}{\partial x_{k}}-\left|\nabla u_{i}^{*}\right|^{p-2} \frac{\partial u_{i}^{*}}{\partial x_{k}}\right)_{k=1}^{m} d x \\
=\int_{\Omega} \nabla \cdot\left(\left|u_{i}-u_{i}^{*}\right|^{p-2}\left(u_{i}-u_{i}^{*}\right)\left(\left|\nabla u_{i}\right|^{p-2} \frac{\partial u_{i}}{\partial x_{k}}-\left|\nabla u_{i}^{*}\right|^{p-2} \frac{\partial u_{i}^{*}}{\partial x_{k}}\right)_{k=1}^{m}\right) d x \\
-\int_{\Omega}\left(\left|\nabla u_{i}\right|^{p-2} \frac{\partial u_{i}}{\partial x_{k}}-\left|\nabla u_{i}^{*}\right|^{p-2} \frac{\partial u_{i}^{*}}{\partial x_{k}}\right)_{k=1}^{m} \nabla\left(\left|u_{i}-u_{i}^{*}\right|^{p-2}\left(u_{i}-u_{i}^{*}\right)\right) d x
\end{aligned}
$$




$$
\begin{aligned}
= & \int_{\partial \Omega}\left(\left|u_{i}-u_{i}^{*}\right|^{p-2}\left(u_{i}-u_{i}^{*}\right)\left(\left|\nabla u_{i}\right|^{p-2} \frac{\partial u_{i}}{\partial x_{k}}-\left|\nabla u_{i}^{*}\right|^{p-2} \frac{\partial u_{i}^{*}}{\partial x_{k}}\right)_{k=1}^{m}\right) \cdot \vec{n} d s \\
& -\int_{\Omega}(p-1)\left|u_{i}-u_{i}^{*}\right|^{p-2} \nabla\left(u_{i}-u_{i}^{*}\right) \cdot\left(\left|\nabla u_{i}\right|^{p-2} \frac{\partial u_{i}}{\partial x_{k}}-\left|\nabla u_{i}^{*}\right|^{p-2} \frac{\partial u_{i}^{*}}{\partial x_{k}}\right)_{k=1}^{m} d x \\
= & -\sum_{k=1}^{m} \int_{\Omega}(p-1)\left|u_{i}-u_{i}^{*}\right|^{p-2}\left(\left|\nabla u_{i}\right|^{p-2} \frac{\partial u_{i}}{\partial x_{k}}-\left|\nabla u_{i}^{*}\right|^{p-2} \frac{\partial u_{i}^{*}}{\partial x_{k}}\right) \cdot\left(\frac{\partial u_{i}}{\partial x_{k}}-\frac{\partial u_{i}^{*}}{\partial x_{k}}\right) d x \\
\leq & 0
\end{aligned}
$$

where $\vec{n}$ is unit outer cotangent vector on $\partial \Omega$. By (3.4), (3.5), (H1), and Young's inequality, one has that

$$
\begin{aligned}
d \overline{V_{i}(u)} \leq & -\sum_{k=1}^{m} \int_{\Omega}(p-1)\left|u_{i}-u_{i}^{*}\right|^{p-2}\left(\left|\nabla u_{i}\right|^{p-2} \frac{\partial u_{i}}{\partial x_{k}}-\left|\nabla u_{i}^{*}\right|^{p-2} \frac{\partial u_{i}^{*}}{\partial x_{k}}\right) \cdot\left(\frac{\partial u_{i}}{\partial x_{k}}-\frac{\partial u_{i}^{*}}{\partial x_{k}}\right) d x \\
& +\left(-p a_{i}+p(p-1) \lambda_{i}\right)\left\|u_{i}(t)-u_{i}^{*}\right\|_{p}^{p} d t \\
& +p \sum_{j=1}^{n} \int_{G}\left|u_{i}-u_{i}^{*}\right|^{p-2}\left|u_{i}-u_{i}^{*}\right|\left|T_{i j}\right| c_{j}\left|u_{j}\left(t-\tau_{j}, x\right)-u_{j}^{*}\right| d x d t \\
& +p \sum_{l=1}^{m} \int_{G}\left|u_{i}-u_{i}^{*}\right|^{p-2}\left(u_{i}-u_{i}^{*}\right)\left(\sigma_{i l}\left(u_{i}\right)-\sigma_{i l}\left(u_{i}^{*}\right)\right) d x d W_{l}(t) \\
\leq & \sum_{j=1}^{n} p_{i j}\left\|u_{i}(t)-u_{i}^{*}\right\|_{p}^{p} d t+\sum_{j=1}^{n} q_{i j}\left\|u_{j}\left(t-\tau_{j}, x\right)-u_{j}^{*}\right\|_{p}^{p} d t \\
& +p \sum_{l=1}^{m} \int_{G}\left|u_{i}-u_{i}^{*}\right|^{p-2}\left(u_{i}-u_{i}^{*}\right)\left(\sigma_{i l}\left(u_{i}\right)-\sigma_{i l}\left(u_{i}^{*}\right)\right) d x d W_{l}(t)
\end{aligned}
$$

where $p_{i j}$ and $q_{i j}$ are defined by (3.1).

For $\Delta t>0$, both sides of (3.6) are integrated about $t$ from $t$ to $t+\Delta t$, then both sides of (3.6) are calculated expectation. By the properties of Brownian motion, one has that

$$
\begin{aligned}
& E\left[\left\|u_{i}(t+\Delta t)-u_{i}^{*}\right\|_{p}^{p}\right]-E\left[\left\|u_{i}(t)-u_{i}^{*}\right\|_{p}^{p}\right] \\
& \quad \leq \sum_{j=1}^{n}\left\{p_{i j} E\left[\int_{t}^{t+\Delta t}\left\|u_{i}(s)-u_{i}^{*}\right\|_{p}^{p} d s\right]+q_{i j} E\left[\int_{t}^{t+\Delta t}\left\|u_{j}\left(s-\tau_{j}(s)\right)-u_{j}^{*}\right\|_{p}^{p} d s\right]\right\} .
\end{aligned}
$$


Since the integrals $\int_{t}^{t+\Delta t} E\left[\left\|u_{i}(s)-u_{i}^{*}\right\|_{p}^{p}\right] d s$ and $\int_{t}^{t+\Delta t} E\left[\left\|u_{j}\left(s-\tau_{j}\right)-u_{i}^{*}\right\|_{p}^{p}\right] d s$ are finite, by Fubini theorem [18] and (3.7), one obtain that

$$
\begin{aligned}
E\left[\left\|u_{i}(t+\Delta t)-u_{i}^{*}\right\|_{p}^{p}\right]-E\left[\left\|u_{i}(t)-u_{i}^{*}\right\|_{p}^{p}\right] \leq & \sum_{j=1}^{n} p_{i j} \int_{t}^{t+\Delta t} E\left[\left\|u_{i}(s)-u_{i}^{*}\right\|_{p}^{p}\right] d s \\
& +\sum_{j=1}^{n} q_{i j} \int_{t}^{t+\Delta t} E\left[\left\|u_{j}\left(s-\tau_{j}(s)\right)-u_{j}^{*}\right\|_{p}^{p}\right] d s .
\end{aligned}
$$

Set $v_{i}(t)=E\left[\left\|u_{i}(t)-u_{i}^{*}\right\|_{p}^{p}\right]$. Both sides of Inequality (3.8) are divided by $\Delta t$, let $\Delta t \rightarrow 0$, one has the following inequality:

$$
D^{+} v_{i}(t) \leq \sum_{j=1}^{n}\left[p_{i j} v_{i}(t)+q_{i j} v_{j}\left(t-\tau_{j}(t)\right)\right]
$$

By Lemma 2.4, there are positive constants $K_{i}, \alpha$ such that

$$
E\left[\left\|u_{i}(t)-u_{i}^{*}\right\|_{p}^{p}\right] \leq K_{i} \sum_{j=1}^{n}\left\|E\left[\left\|\phi_{j}-u_{j}^{*}\right\|_{p}^{p}\right]\right\| \exp \left(-\alpha\left(t-t_{0}\right)\right), \quad i=1, \ldots, n, t \geq t_{0},
$$

where $\phi_{j}$ is initial value. Set $K=\max \left\{K_{i}: 1 \leq i \leq n\right\}$, then

$$
E\left[\sum_{j=1}^{n}\left\|u_{j}-u_{j}^{*}\right\|_{p}^{p}\right] \leq n K \sum_{j=1}^{n}\left\|E\left[\left\|\phi_{j}-u_{j}^{*}\right\|_{p}^{p}\right]\right\| \exp \left(-\alpha\left(t-t_{0}\right)\right), \quad t \geq t_{0} .
$$

By (3.11) and Lemma 2.5, one obtains that

$$
E\left[\left\|u-u^{*}\right\|_{p}^{p}\right] \leq e_{p}^{-1}(n) d_{p}(n) n K \sum_{j=1}^{n}\left\|E\left[\left\|\phi_{j}-u_{j}^{*}\right\|_{p}^{p}\right]\right\| \exp \left(-\alpha\left(t-t_{0}\right)\right), \quad t \geq t_{0} .
$$

In order to prove Theorem 3.1, we need the following lemma.

Lemma 3.2. The nonconstant equilibrium solution of the problem (1.1)-(1.3), $u^{*}(x)$ satisfies $E\left(\left\|u^{*}\right\|_{p}^{p}\right)=0$.

Proof. Set $F_{i}\left(u^{*}(x)\right)=\left|u_{i}^{*}(x)\right|^{p}$. Similar to (3.8) in proof of Theorem 3.1, one has that

$$
\sum_{j=1}^{n} p_{i j} \int_{t}^{t+\Delta t} E\left[\left\|u_{i}^{*}\right\|_{p}^{p}\right] d s+\sum_{j=1}^{n} q_{i j} \int_{t}^{t+\Delta t} E\left[\left\|u_{j}^{*}\right\|_{p}^{p}\right] d s \geq 0
$$


By (3.13) and the assumption that $-\left(p_{j i}+q_{i j}\right)_{n \times n}:=P^{T}+Q$ is an M-matrix, one obtains that

$$
-\sum_{j=1}^{n}\left(p_{j i}+q_{i j}\right) E\left[\left\|u_{j}^{*}\right\|_{p}^{p}\right] \Delta t \leq 0, \quad i=1,2, \ldots, n .
$$

Because of $E\left[\left\|u_{j}^{*}\right\|_{p}^{p}\right] \geq 0$ and $\Delta t>0$, one has that $E\left(\left\|u^{*}\right\|_{p}^{p}\right)=0$.

We continue the proof of Theorem 3.1 as the following.

By Lemma 3.2, one has that

$$
\left\|E\left[\left\|\phi_{j}-u_{j}^{*}\right\|_{p}^{p}\right]\right\| \leq M
$$

where $M>0$ is a common number. We derive every solution of problem (1.1)-(1.3) such that

$$
\limsup _{t \rightarrow \infty} \frac{1}{t} \log E\left[\left\|u-u^{*}\right\|_{p}^{p}\right] \leq-\alpha
$$

then a nonconstant equilibrium solution of problem (1.1)-(1.3) about $L^{p}$ norm is exponential stability in $p$ th moment. The proof of Theorem 3.1 is complete.

In order to illustrate the application of the theorem, we give an example.

Example 3.3. Discuss the stochastic reaction-diffusion neural network with time-varying delays and $p$-Laplacian as the following:

$$
\begin{aligned}
& d u_{1}(t, x)= {\left[\sum_{k=1}^{2} \frac{\partial}{\partial x_{k}}\left(\left|\nabla u_{1}\right|^{p-2} \frac{\partial u_{1}}{\partial x_{k}}\right)-a_{1} u_{1}+I_{1}+\sum_{j=1}^{2} T_{1 j} g_{j}\left(u_{j}\left(t-\tau_{j}(t), x\right)\right)\right] d t } \\
&+\sigma_{1}\left(u_{1}(t, x)\right) d W(t), \\
& d u_{2}(t, x)= {\left[\sum_{k=1}^{2} \frac{\partial}{\partial x_{k}}\left(\left|\nabla u_{2}\right|^{p-2} \frac{\partial u_{2}}{\partial x_{k}}\right)-a_{2} u_{2}+I_{2}+\sum_{j=1}^{2} T_{2 j} g_{j}\left(u_{j}\left(t-\tau_{j}(t), x\right)\right)\right] d t } \\
&+\sigma_{2}\left(u_{2}(t, x)\right) d W(t), \\
& \frac{\partial u_{i}}{\partial n}:=\left(\frac{\partial u_{i}}{\partial x_{1}}, \frac{\partial u_{i}}{\partial x_{2}}\right)^{T}=0, \quad i=1,2, t \geq t_{0} \geq 0, x \in \partial \Omega, \\
& u_{i}\left(t_{0}+s, x\right)=\phi_{i}(s, x), \quad-\tau_{i}\left(t_{0}\right) \leq s \leq 0,0 \leq \tau_{i}(t) \leq \tau_{i}, i=1,2, x \in \Omega,
\end{aligned}
$$


where

$$
\begin{gathered}
a_{1}=1.2, \quad a_{2}=1.8, \quad \sigma_{1}\left(u_{1}\right)=0.2\left(u_{1}-u_{1}^{*}\right), \quad \sigma_{2}\left(u_{2}\right)=0.3\left(u_{2}-u_{2}^{*}\right), \\
T_{11}=1.5, \quad T_{12}=0.5, \quad T_{21}=0.6, \quad T_{22}=1, \\
g_{1}\left(u_{1}\left(t-\tau_{1}(t), x\right)\right)=0.3 u_{1}\left(t-\tau_{1}(t), x\right) \\
g_{2}\left(u_{2}\left(t-\tau_{2}(t), x\right)\right)=0.2 u_{2}\left(t-\tau_{2}(t), x\right) \cos \left(u_{2}\left(t-\tau_{2}(t), x\right)\right) .
\end{gathered}
$$

Set $u^{*}(x)=\left(u_{1}^{*}(x), u_{1}^{*}(x)\right)^{T}$ as a nonconstant equilibrium solution of (3.17) and (3.18). One can derive that

$$
\begin{aligned}
&\left|g_{1}\left(v_{1}\right)-g_{1}\left(v_{2}\right)\right| \leq 0.3\left|v_{1}-v_{2}\right|, \quad c_{1}=0.3 \\
&\left|g_{2}\left(v_{1}\right)-g_{2}\left(v_{2}\right)\right| \leq 0.4\left|v_{1}-v_{2}\right|, \quad c_{2}=0.4 ; \\
&\left|\left(\sigma_{1}\left(v_{1}\right)-\sigma_{1}\left(v_{2}\right)\right)\left(\sigma_{1}\left(v_{1}\right)-\sigma_{1}\left(v_{2}\right)\right)^{T}\right| \leq 0.04\left|v_{1}-v_{2}\right|, \quad \lambda_{1}=0.02 \\
&\left|\left(\sigma_{2}\left(v_{1}\right)-\sigma_{2}\left(v_{2}\right)\right)\left(\sigma_{2}\left(v_{1}\right)-\sigma_{2}\left(v_{2}\right)\right)^{T}\right| \leq 0.09\left|v_{1}-v_{2}\right|, \quad \lambda_{2}=0.045
\end{aligned}
$$

Taking $\varepsilon_{i}=1(i=1,2)$ and $p=3$, one has that

$$
M_{3}=\left(\begin{array}{cc}
2.58 & -0.56 \\
-0.58 & 1.53
\end{array}\right)
$$

and $M_{3}$ is an $M$-matrix. The nonconstant equilibrium solution of (3.17) and (3.18) about $L^{3}$ norm is exponential stability in the 3rd moment.

Remark 3.4. The Theorem 3.1 extends the correlative results in $[12,13,16]$ to the situation related to the $p$-Laplacian.

\section{Acknowledgments}

The authors thank the reviewers for their constructive comments. This work is supported by the National Science Foundation of China (no. 10971240).

\section{References}

[1] X. Liao and J. Wang, "Global dissipativity of continuous-time recurrent neural networks with time delay," Physical Review E, vol. 68, no. 1, Article ID 016118, 6 pages, 2003.

[2] L. Wang and D. Xu, "Stability for Hopfield neural networks with time delay," Journal of Vibration and Control, vol. 8, no. 1, pp. 13-18, 2002.

[3] D. Xu, H. Zhao, and H. Zhu, "Global dynamics of Hopfield neural networks involving variable delays," Computers \& Mathematics with Applications, vol. 42, no. 1-2, pp. 39-45, 2001.

[4] L. S. Wang and D. Y. Xu, "Stability analysis of Hopfield neural networks with time delay," Applied Mathematics and Mechanics, vol. 23, no. 1, pp. 65-70, 2002.

[5] X. Liao, X. Mao, J. Wang, and Z. Zeng, "Algebraic conditions of stability for Hopfield neural network," Science in China F, vol. 47, no. 1, pp. 113-125, 2004. 
[6] X. X. Liao, S. Z. Yang, S. J. Cheng, and Y. Shen, "Stability of general neural networks with reactiondiffusion," Sciena in China E, vol. 32, no. 1, pp. 87-94, 2002 (Chinese).

[7] L. Wang and D. Xu, "Global exponential stability of Hopfield reaction-diffusion neural networks with time-varying delays," Science in China F, vol. 46, no. 6, pp. 466-474, 2003.

[8] S. Blythe, X. Mao, and X. Liao, "Stability of stochastic delay neural networks," Journal of the Franklin Institute, vol. 338, no. 4, pp. 481-495, 2001.

[9] S. Hu, X. Liao, and X. Mao, "Stochastic Hopfield neural networks," Journal of Physics A, vol. 36, pp. 2235-2249, 2003.

[10] J. R. Niu, Z. F. Zhang, and D. Y. Xu, "Exponential stability in mean square of a stochastic CohenGrossberg neural network with time-varying delays," Chinese Journal of Engineering Mathematics, vol. 22, no. 6, pp. 1001-1005, 2005 (Chinese).

[11] Y. Shen, M. H. Jiang, and H. S. Yao, "Exponential stability of cellular neural networks," Acta Mathematica Scientia A, vol. 25, no. 2, pp. 264-268, 2005 (Chinese).

[12] Q. Luo, F. Deng, J. Bao, B. Zhao, and Y. Fu, "Stabilization of stochastic Hopfield neural network with distributed parameters," Science in China F, vol. 47, no. 6, pp. 752-762, 2004.

[13] Z. Zifang, The stability of uncertain dynamical systems and applications [M.S. thesis], Sichuan University, 2004.

[14] S. J. Long and L. Xiang, "A nonlinear measure method for stability of Hopfield neural networks with time-varying delays," Journal of Sichuan University, vol. 44, no. 2, pp. 249-252, 2007 (Chinese).

[15] Z.-F. Zhang and D.-Y. Xu, "A note on stability of stochastic delay neural networks," Chinese Journal of Engineering Mathematics, vol. 27, no. 4, pp. 720-730, 2010.

[16] Z. F. Zhang and J. Deng, "Stability of stochastic reaction-diffusion Hopfield neural network with time-varying delays," Journal of Sichuan University, vol. 47, no. 3, pp. 251-256, 2010.

[17] D. Xu, "Stability criteria of large-scale systems of the neutral type," in Proceedings of the12th IMACS World Congress on Scientific Computations, R. Vichnevetsky, P. Borne, and J. Vignes, Eds., vol. 1, p. 213, Gerfidn-cite Scientifique, Pairs, France, 1988.

[18] W. Rudin, Real and Complex Analysis, McGraw-Hill, 1974. 


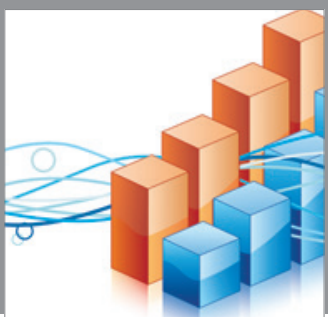

Advances in

Operations Research

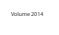

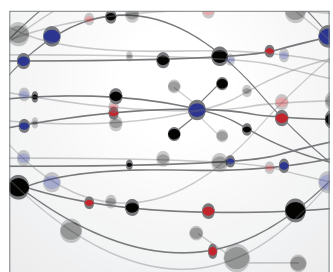

\section{The Scientific} World Journal
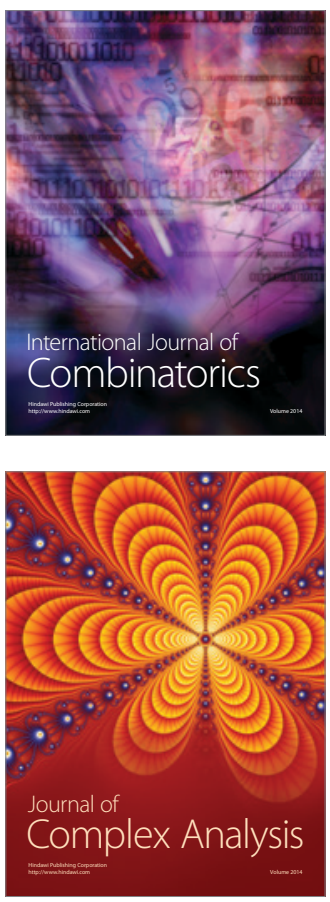

International Journal of

Mathematics and

Mathematical

Sciences
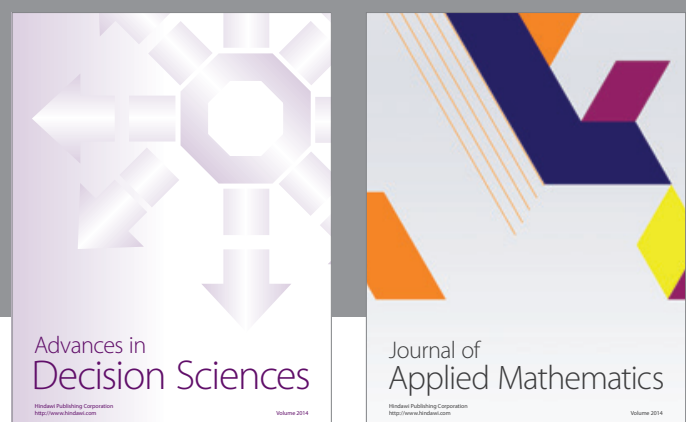

Journal of

Applied Mathematics
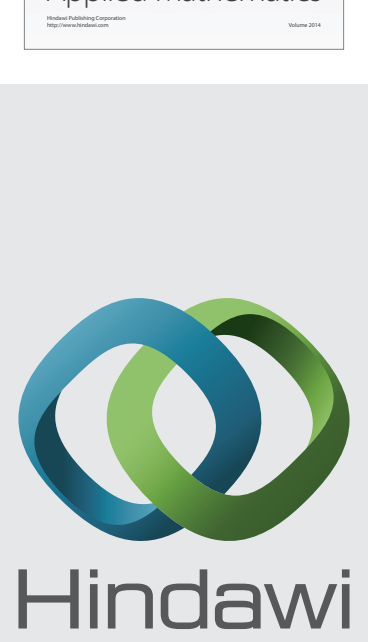

Submit your manuscripts at http://www.hindawi.com
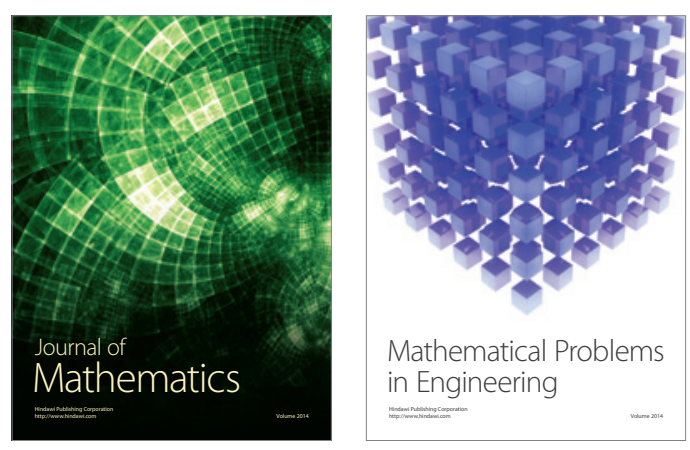

Mathematical Problems in Engineering
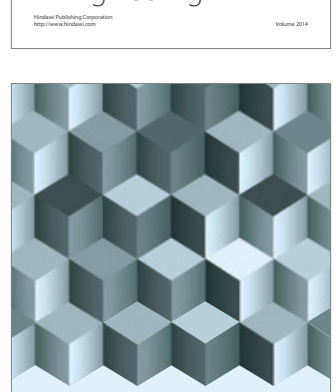

Journal of

Function Spaces
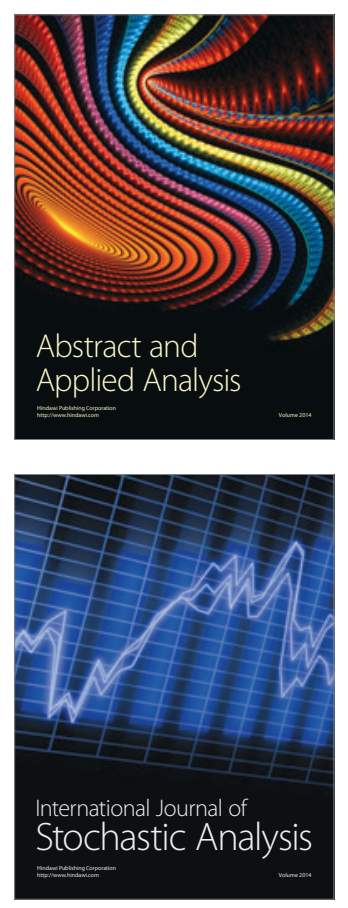

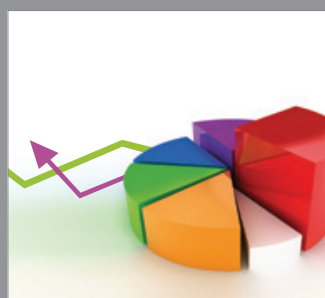

ournal of

Probability and Statistics

Promensencen
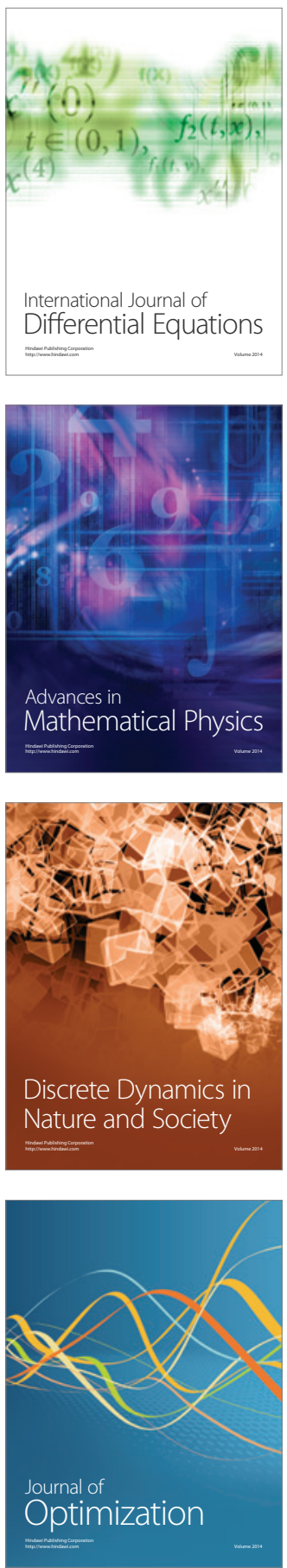